\title{
Optimized Large Hyperuniform Binary Colloidal Suspensions in Two Dimensions
}

\author{
Zheng Ma \\ Department of Physics, Princeton University, Princeton, New Jersey 08544, USA \\ Enrique Lomba \\ Instituto de Química Física Rocasolano, CSIC, Calle Serrano 119, E-28006 Madrid, Spain \\ Salvatore Torquato** \\ Department of Chemistry, Department of Physics, Princeton Institute for the Science and Technology of Materials, \\ and Program in Applied and Computational Mathematics, Princeton University, Princeton, New Jersey 08544, USA
}

(Received 30 March 2020; accepted 15 July 2020; published 7 August 2020)

\begin{abstract}
The creation of disordered hyperuniform materials with extraordinary optical properties (e.g., large complete photonic band gaps) requires a capacity to synthesize large samples that are effectively hyperuniform down to the nanoscale. Motivated by this challenge, we propose a feasible equilibrium fabrication protocol using binary paramagnetic colloidal particles confined in a 2D plane. The strong and long-ranged dipolar interaction induced by a tunable magnetic field is free from screening effects that attenuate long-ranged electrostatic interactions in charged colloidal systems. Specifically, we numerically find a family of optimal size ratios that makes the two-phase system effectively hyperuniform. We show that hyperuniformity is a general consequence of low isothermal compressibilities, which makes our protocol suitable to treat more general systems with other long-ranged interactions, dimensionalities, and/ or polydispersity. Our methodology paves the way to synthesize large photonic hyperuniform materials that function in the visible to infrared range and hence may accelerate the discovery of novel photonic materials.
\end{abstract}

DOI: 10.1103/PhysRevLett.125.068002

Introduction.-Disordered hyperuniform systems are exotic disordered states that lie between crystals and liquids. While being statistically isotropic like liquids, they exhibit "hidden order" in the sense that they suppress largescale density fluctuations in the way that crystals do. Since the fundamental study on this subject [1], hyperuniformity has been observed in a surprisingly wide variety of systems. Examples range from classical equilibrium systems [2-4], classical and quantum jammed systems [5-8], critical absorbing states [9-11], active matter [12,13], soft polymers [14], biological systems $[15,16]$, to the onedimensional point patterns derived from the nontrivial zeros of the Riemann zeta function [17]; see also the recent review for a more comprehensive list [18]. Hyperuniform point configurations in $d$-dimensional space $\mathbb{R}^{d}$ possess a structure factor $S(\mathbf{k})$ that goes to zero as the wave number $|\mathbf{k}|$ vanishes, i.e., $\lim _{|\mathbf{k}| \rightarrow 0} S(\mathbf{k})=0$, which corresponds to a local number variance $\sigma_{N}^{2}(R)$ in a spherical window of radius $R$ that grows slower than $R^{d}$. The hyperuniformity concept has been generalized to two-phase media [19], where hyperuniformity means that the spectral density $\tilde{\chi}_{V}(\mathbf{k})$ (Fourier transform of the autocovariance function [20]) goes to zero as $|\mathbf{k}| \rightarrow \mathbf{0}$, which is equivalent to a local volume-fraction variance $\sigma_{V}^{2}(R)$ that decreases faster than $R^{-d}$ for large $R$.
Besides being of great fundamental interest, hyperuniform materials are showing exciting technological promise, especially in photonics [21-27]. Specifically, disordered hyperuniform dielectric networks can have large and complete photonic band gaps, like photonic crystals, but possess the advantage of being isotropic, enabling freeform waveguides [22]. These studies have spurred the exploration of these unusual materials for acoustic applications [28]. Moreover, hyperuniform materials may be useful for producing vivid noniridescent structural colors $[29,30]$. However, a fundamental challenge is how to make large hyperuniform samples efficiently, especially down to the nanoscale. Computational protocols, such as the extensively used collective-coordinate optimization technique [4], involve high computational costs, which makes it difficult to generate sample sizes beyond thousands of particles. Thus, a bottom-up, self-assembly based fabrication method is highly desired. Notable examples include jamming of hard or soft particles [5,6,31,32], periodically driven systems going through an absorbing phase transition $[10,33,34]$, and spinodal decomposition or dewetting $[35,36]$. The fact that many of these hyperuniform nonequilibrium systems are at critical points implies that there is little room to tune the structure, e.g., it is impossible to demand a significantly lower volume fraction in jammed 
systems. Moreover, any imperfections or defects, which always occur in experiments, can degrade hyperuniformity, e.g., rattlers in jammed packings [37].

On the other hand, equilibrium systems are much more robust and flexible in the sense that their macroscopic properties are time independent and can be tuned by many parameters. Specifically, in order to observe hyperuniformity in an equilibrium system, the isothermal compressibility relation $S\left(0^{+}\right)=\rho k_{B} T \kappa_{T}$ dictates a vanishing compressibility $\kappa_{T}$ at positive temperature $T$. This relation implies that a long-ranged interaction must be present. While systems that utilize Coulombic interactions may at first glance appear to be suitable to achieve hyperuniformity (e.g., charged colloids), they often suffer from screening effects that attenuate the associated long-range electrostatic interactions. It has been shown that such systems only become effectively hyperuniform [38] at low temperatures and small inverse screening lengths.

In this Letter, we propose a highly feasible and robust equilibrium protocol that can be used in the laboratory to fabricate large disordered hyperuniform materials down to the nanoscale. Specifically, we consider superparamagnetic colloidal particles (doped with magnetic materials) confined at a two-dimensional (2D) interface such that dipole-dipole interactions are induced when a magnetic field is applied perpendicular to the plane. The interactions in such systems are strong, long-ranged $\left[u(r) \sim 1 / r^{3}\right]$, and free of screening effects, making them excellent candidate systems that can yield large, effectively disordered hyperuniform colloidal systems at positive temperatures. We employ binary particle mixtures to frustrate crystallization [39]. The monodisperse version of our model has been extensively studied both numerically and experimentally [40-43], mainly for probing the nature of 2D melting [44]. Much less is known about the polydisperse case. In this work, we apply both Monte Carlo simulations and integral-equation formalisms to study the structure of the system. Similar techniques were applied in Ref. [45], but hyperuniformity was not a consideration. Moreover, here we focus on the twophase systems formed by the particles, which is crucial for our purposes, as detailed below.

Our main finding is that in the equilibrium liquid phase, despite the structure of each component not being hyperuniform, there exists an optimal size ratio $R_{1} / R_{2}=$ $\left[\rho_{2} S_{22}\left(0^{+}\right) / \rho_{1} S_{11}\left(0^{+}\right)\right]^{(1 / 4)}$ that makes the resulting twophase system effectively hyperuniform. Here $S_{i i}$ and $\rho_{i}$ are the partial structure factor and density of the species $i$. The optimal size ratio leverages the destructive interference between scattering events between the two species, which is a general direct consequence of low isothermal compressibilities due to the strong and long-ranged repulsion. Our protocol can be potentially applied to other longranged soft repulsions $\left[u(r) \sim 1 / r^{n}\right]$, as they are shown to bear similar physics [41]. Additionally, our protocol is also suitable to systems with other dimensionalities and/or polydispersity.

Methods.-We study binary superparamagnetic colloidal particles confined in a 2D plane $[39,45,46]$. An external magnetic field B perpendicular to the plane is applied, inducing a dipole-dipole interaction. For the binary system we consider here, $N_{i}, \chi_{i}, \rho_{i}$, and $x_{i}$ denote the particle number, susceptibility, number density, and concentration for each component, $i=1,2$, respectively, while $\rho$ is the total number density. Species 1 and 2 consist of "large" and "small" particles, respectively, in the sense that $\chi_{1}>\chi_{2}$. The dipole-dipole interaction $u_{i j}$ can be rewritten as $\beta u_{i j}(d)=\Gamma_{i j} / d^{3}$, where $\beta=1 /\left(k_{B} T\right)$, with $k_{B}$ being Boltzmann's constant, and $x$ is the distance between two particles rescaled by the average interparticle distance between large particles, i.e., $d \equiv r / a_{11}$, where $a_{11}=1 / \sqrt{\rho_{1}}$. The quantity $\Gamma_{i j}$ is a dimensionless coupling strength between species $i$ and $j$, which can be written as $\Gamma_{i j}=\beta \mu_{0} \chi_{i} \chi_{j} B^{2} /\left(8 \pi a_{11}^{3}\right)$. The binary colloids have radii $R_{1}$ and $R_{2}$, respectively, however in our simulations they can be treated conveniently as pointlike due to the strong dipole-dipole repulsion [46]. We perform Monte Carlo simulations of a system that consists of 3600 particles, using a simplified swap Monte Carlo algorithm [47]. Up to $10^{9}$ steps were used to equilibrate the systems, and all results presented are averaged using 30 to 50 configurations. The particle sizes are chosen such that the volume fraction is fixed at 0.15 . We also solve the Ornstein-Zernike integral equation numerically to obtain the partial structure factors using the Rogers-Young [48] (RY) closure, see Ref. [49] and Supplemental Material (SM) [50] for details of the corresponding algorithm.

Results.-Importantly, we first show the binary system is not hyperuniform as a point pattern, but there is a unique way of decorating the points with spheres so that this resulting two-phase system is effectively hyperuniform. As noted above, hyperuniformity, i.e., the vanishing of $S\left(0^{+}\right)$ of an equilibrium one-component system, is directly related to its incompressibility. However, we need to consider the isothermal compressibility $\kappa_{T}$ of a binary system, which, in any Euclidean space dimension $d$, can be expressed in terms of the partial structure factors $S_{i j}(k)$ [51]:

$$
\rho k_{B} T \kappa_{T}=\frac{S_{11}\left(0^{+}\right) S_{22}\left(0^{+}\right)-S_{12}^{2}\left(0^{+}\right)}{x_{1} S_{22}\left(0^{+}\right)+x_{2} S_{11}\left(0^{+}\right)-2 x_{1}^{\frac{1}{2}} x_{2}^{\frac{1}{2}} S_{12}\left(0^{+}\right)},
$$

where $S_{i j}(k)=\left(N_{i} N_{j}\right)^{-(1 / 2)}\left\langle\tilde{n}_{i}(k) \tilde{n}_{j}^{*}(k)\right\rangle$. Here $\tilde{n}_{1}(\mathbf{k})$ and $\tilde{n}_{2}(\mathbf{k})$ are complex collective density variables for large and small particles, which are defined as $\tilde{n}_{1}(\mathbf{k}) \equiv \sum_{i=1}^{N_{1}} e^{-i \mathbf{k} \cdot \mathbf{r}_{1 i}}$ and $\tilde{n}_{2}(\mathbf{k}) \equiv \sum_{i=1}^{N_{2}} e^{-i \mathbf{k} \cdot \mathbf{r}_{2 i}}$, where $\left\{r_{1 i}\right\}$ and $\left\{r_{2 i}\right\}$ refer to the set of positions of large and small particles. Incompressibility $\left(\kappa_{T}=0\right)$ at positive temperature then implies that 


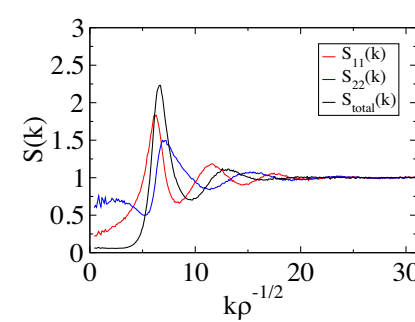

(a) $\Gamma_{22}=1$

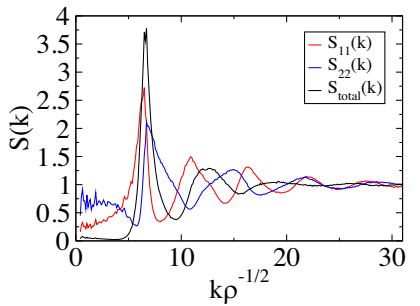

(c) $\Gamma_{22}=5$

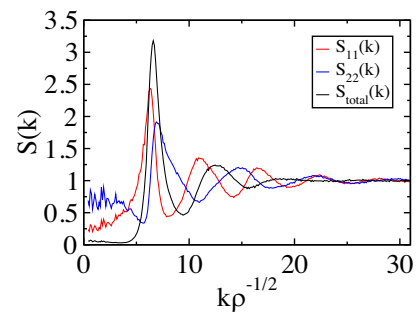

(b) $\Gamma_{22}=3$

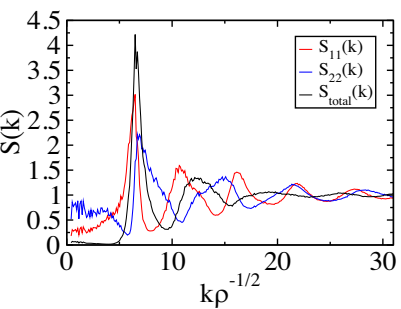

(d) $\Gamma_{22}=10$

FIG. 1. Structure factors for large particles $S_{11}(k)$, small particles $S_{22}(k)$, and the entire system $S_{\text {total }}(k)$ for different values of the coupling strength: (a) $\Gamma_{22}=1$, (b) $\Gamma_{22}=3$, (c) $\Gamma_{22}=5$, (d) $\Gamma_{22}=10$. Clearly, neither the point pattern associated with each component nor the entire system is hyperuniform.

$$
S_{11}\left(0^{+}\right) S_{22}\left(0^{+}\right)-S_{12}^{2}\left(0^{+}\right)=0 \text {. }
$$

While incompressibility cannot be perfectly achieved in the dipolar system we study, due to the strong and long-ranged repulsion, this condition will approximately hold when the coupling strength $\Gamma$ is sufficiently large.

However, we show that incompressibility in our equilibrium binary systems means the destructive interference of scattering events between both species, rather than the vanishing of $S(k)$ itself as the case in monodisperse systems. To demonstrate this idea, we present simulation results of a binary system with the number density ratio $\rho_{1} / \rho_{2}=1$ and the susceptibility ratio $\chi_{1} / \chi_{2}=2$. Figure 1 depicts the computed structure factors for the small particles $S_{22}(k)$, large particles $S_{11}(k)$, and the entire system $S_{\text {total }}(k)$ for different coupling strengths. Here $S_{\text {total }}(k)$ can be written as $N^{-1}\left\langle\left|\tilde{n}_{i}(k)+\tilde{n}_{j}(k)\right|^{2}\right\rangle$.

Clearly, neither the point pattern associated with each component or the entire system of points is hyperuniform, which requires vanishing structure factors when $k$ goes to zero. Interestingly, we find that compared to the structure factors of each component, the small- $k$ values of the structure factor $S_{\text {total }}(k)$ are suppressed, while the first peak is significantly enhanced. The $k$ value of the first intersection of $S_{11}(k)$ and $S_{22}(k)$ corresponds to the minimum of $S_{\text {total }}(k)$, which decreases as the coupling strength increases. These facts suggest that the interference due to scattering from the small and large particles is constructive near the wavelength associated with the first peak of the structure factor, while destructive in the small- $k$

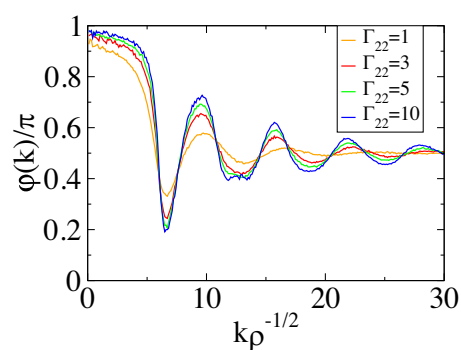

(a)

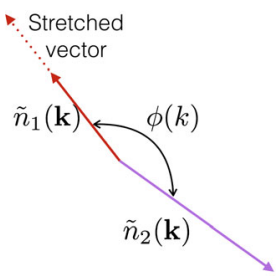

(b)
FIG. 2. (a) The angle $\phi(k)$ between the complex collective density variables for small particles $\tilde{n}_{2}(\mathbf{k})$ and large particles $\tilde{n}_{1}(\mathbf{k})$ as a function of wave number $k$ under different coupling strengths. (b) A schematic plot of the complex collective density variables for small particles $\tilde{n}_{2}(\mathbf{k})$ and large particles $\tilde{n}_{1}(\mathbf{k})$ in the complex plane. Here, vector $\tilde{n}_{1}(\mathbf{k})$ can be stretched out to cancel $\tilde{n}_{2}(\mathbf{k})$ when they are added together.

region, which favors hyperuniformity. To demonstrate this observation quantitatively, we compute the angle $\phi(\mathbf{k})$ between $\tilde{n}_{1}(\mathbf{k})$ and $\tilde{n}_{2}(\mathbf{k})$, defined by

$$
\begin{aligned}
& \phi(\mathbf{k}) \\
& =\arccos \frac{\operatorname{Re}\left(\tilde{n}_{1}(\mathbf{k})\right) \operatorname{Re}\left(\tilde{n}_{2}(\mathbf{k})\right)+\operatorname{Im}\left(\tilde{n}_{1}(\mathbf{k})\right) \operatorname{Im}\left(\tilde{n}_{2}(\mathbf{k})\right)}{\left|\tilde{n}_{1}(\mathbf{k})\right|\left|\tilde{n}_{2}(\mathbf{k})\right|} .
\end{aligned}
$$

We plot the angular-averaged $\phi(k)$ as a function of wave number $k$ in Fig. 2(a). These results justify our previous arguments, i.e., in the small- $k$ region, the angle $\phi(k)$ is very close to $\pi$, showing that the two complex collective density variables align themselves in opposite directions and thus the interference is destructive [see schematic shown in Fig. 2(b)]; while the first dip of $\phi(k)$ coincides with the location of the first peak, showing that the interference is constructive at the peak. When $k \rightarrow \infty$, the angle converges to $\pi / 2$, which confirms the expectation that the correlation finally dies out. Importantly, as the coupling strength increases, the two complex collective density variables are more strongly aligned with each other. We now show how this finding relates to Eq. (2). Using the aforementioned complex collective density variables, it easily follows that $S_{12}(k)$ can be written as $\sqrt{S_{11}(k) S_{22}(k)} \cos \phi(k)$. Plugging into Eq. (2), we have

$$
S_{11}\left(0^{+}\right) S_{22}\left(0^{+}\right)\left[1-\cos ^{2} \phi\left(0^{+}\right)\right]=0 .
$$

The solution of Eq. (4) means that $\phi\left(0^{+}\right)=\pi$. Moreover, for dense liquids, numerically we found $S_{11}\left(0^{+}\right)$and $S_{22}\left(0^{+}\right)$are insensitive to $\Gamma$, thus we approximately have $\left[1+\cos \phi\left(0^{+}\right)\right] \propto T \kappa_{T}$ according to Eq. (1). This relation explains the results in Fig. 2(a), i.e., the angle $\phi\left(0^{+}\right)$ converges to $\pi$ as the coupling strength increases.

However, although $\tilde{n}_{1}\left(0^{+}\right)$and $\tilde{n}_{2}\left(0^{+}\right)$almost align themselves in opposite directions in the complex plane, 
due to the fact that their magnitudes are different, their sum as well as the resulting total structure factor $S_{\text {total }}\left(0^{+}\right)$ cannot vanish. This suggests that if we can elongate the vectors $\tilde{n}_{1}\left(0^{+}\right)$and $\tilde{n}_{2}\left(0^{+}\right)$such that their magnitudes match each other, their sum would be very close to zero, as we indicated in Fig. 2(b). Importantly, this idea enables us to design an optimized hyperuniform two-phase system based on decorating the point pattern.

For a two-phase medium, hyperuniformity is defined in terms of the spectral density $\tilde{\chi}_{V}(\mathbf{k})$, as noted earlier. In general, the spectral density can be written as $|\tilde{J}(k)|^{2} / V$, where $\tilde{J}(k)$ is the Fourier transform of $J(\mathbf{x})=I(\mathbf{x})-\langle I(\mathbf{x})\rangle$ and $I(\mathbf{x})$ is the particle-phase indicator function [19]. In the small- $k$ region, we have the following approximate expression for our binary system:

$$
\tilde{J}(k)=\pi R_{1}^{2} \tilde{n}_{1}(k)+\pi R_{2}^{2} \tilde{n}_{2}(k) .
$$

To make $\left|\tilde{J}\left(0^{+}\right)\right|$as close to zero as possible, we immediately come to the relation that $R_{1}^{2}\left|\tilde{n}_{1}\left(0^{+}\right)\right|=R_{2}^{2}\left|\tilde{n}_{2}\left(0^{+}\right)\right|$, which leads to the optimal particle size ratio (see SM for a formal derivation):

$$
\frac{R_{1}}{R_{2}}=\left(\frac{\rho_{2} S_{22}\left(0^{+}\right)}{\rho_{1} S_{11}\left(0^{+}\right)}\right)^{\frac{1}{4}}
$$

Generally, the susceptibility ratio $\chi_{1} / \chi_{2}$ is only dependent on the doping level, thus it can be independent of the size ratio $R_{1} / R_{2}$. This provides great flexibility to tune the system to the optimal hyperuniform state. Observe that in Fig. 1, as the coupling strength increases, the values of the structure factors $S_{11}\left(0^{+}\right)$and $S_{22}\left(0^{+}\right)$approximately remain the same. This insensitivity shows that the optimal particle size ratio is essentially determined by the composition, but not the external field. This is particularly important from an experimental point of view because it means that the optimal colloid composition can be prescribed and one only needs to tune the magnetic field to the desired level.

To demonstrate the effectiveness of our protocol, we consider decorating the simulated point pattern with particles with different size ratios while keeping the volume fraction of the particle phase fixed (here we use 0.15). Figure 3 shows corresponding spectral densities $\tilde{\chi}_{V}(k)$ for these systems. Indeed, we find that the optimal size ratio $(\approx 1.3)$ gives the smallest $\tilde{\chi}_{V}\left(0^{+}\right)$. To quantify how close the system is to perfect hyperuniformity, we employ the "hyperuniformity index," $H$, defined as $H=\tilde{\chi}_{V}\left(0^{+}\right) / \tilde{\chi}_{V}\left(k_{\max }\right)$, where $\tilde{\chi}_{V}\left(k_{\max }\right)$ is the value of the largest peak of the spectral density [37]. We find for $\Gamma_{22}=10$, the hyperuniformity index $H$ is as small as 0.0004 , which is 2 orders of magnitude smaller than the value obtained from the system with identical particle sizes. Moreover, we find empirically that for the optimal structure, the relation $\tilde{\chi}_{V}\left(0^{+}\right) \propto 1 / \Gamma_{22}$ holds, which is very similar to the behavior of the

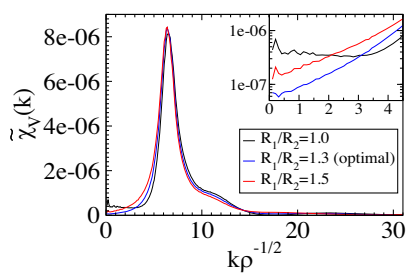

(a) $\Gamma_{22}=1$

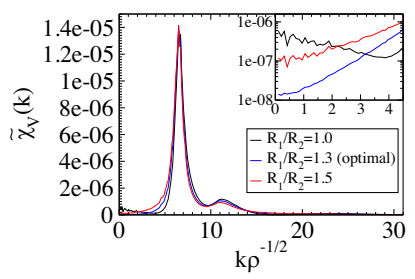

(c) $\Gamma_{22}=5$

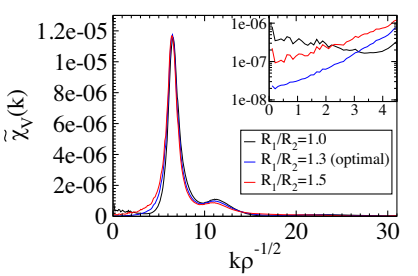

(b) $\Gamma_{22}=3$

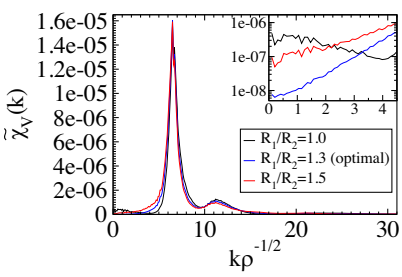

(d) $\Gamma_{22}=10$
FIG. 3. Spectral densities $\tilde{\chi}_{V}(k)$ for binary systems with different size ratios $R_{1} / R_{2}$ for different values of the coupling strength: (a) $\Gamma_{22}=1$, (b) $\Gamma_{22}=3$, (c) $\Gamma_{22}=5$, (d) $\Gamma_{22}=10$. Insets show that the small- $k$ spectral density values for the optimal size ratio is much smaller than those for nonoptimal ratios. For $\Gamma_{22}=10$, the hyperuniformity index $H$ is as small as 0.0004 for the optimal size ratio.

monodisperse structure factors. This interesting similarity between the spectral density $\tilde{\chi}_{V}(k)$ of the binary system with the optimal size ratio and the structure factor of a monodisperse system can be shown by substituting the optimal size ratio into Eq. (5). We find that $\tilde{\chi}_{V}\left(0^{+}\right) \propto$ $2 \pi^{2} R_{1}^{4}\left|\tilde{n}_{1}\left(0^{+}\right)\right|^{2}\left[1+\cos \phi\left(0^{+}\right)\right] \propto T \kappa_{T}$. In the case of dense dipolar systems, this implies that we approximately have $\tilde{\chi}_{V}\left(0^{+}\right) \propto 1 / \Gamma$ [52], which indeed is what we find in simulations, see SM. This finding suggests that we can control the volume-fraction fluctuations at long wavelengths by tuning the magnetic field, while retaining the isotropy of a disordered system. We depict a realization of the optimal system at $\Gamma_{22}=5$ in Fig. 4(a). In Fig. 4(b), we directly compute the local volume-fraction variances $\sigma_{V}^{2}(R)$ associated with windows of radii $R$ [19] for different size ratios. Note that at the optimal size ratio $(\approx 1.3)$, the volume-fraction variance indeed decreases fastest with $R$. Moreover, a scaling of $R^{-2.9}$ is found for the optimal configuration. These results further confirm that our protocol gives the most hyperuniform configuration among all possible size ratios.

We have found similar results for other sets of parameters, including spectral densities and local volume fraction variances for two other binary systems (see the SM for details). To provide a useful recipe, we use the RY approximation to systematically study the manifold of the optimal size ratio as a function of the coupling strength ratio, $\gamma_{12}=\Gamma_{11} / \Gamma_{22}$ and composition. The function $Z\left(x_{1}, \gamma_{12}\right)=$ $R_{1} / R_{2}$ is computed from Eq. (6) where the partial structure factors are determined using the RY approximation. This quantity is plotted in Fig. 4(c). Interestingly, the optimal size ratio is almost independent of the composition as long as $\gamma_{12}$ is not too large. Using the effective hard disk diameter 


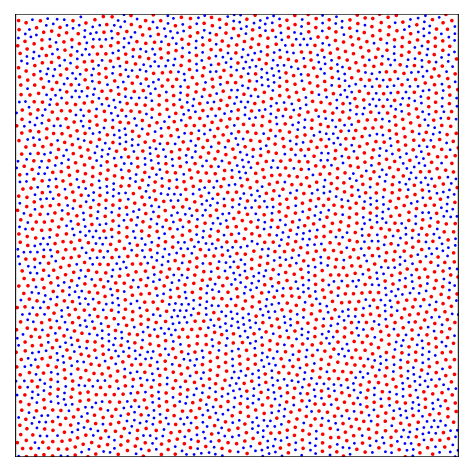

(a)

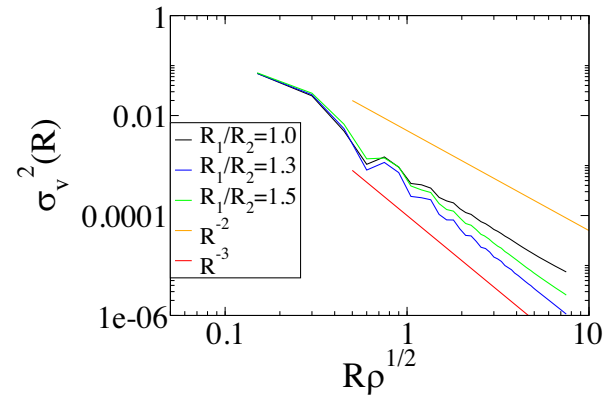

(b)

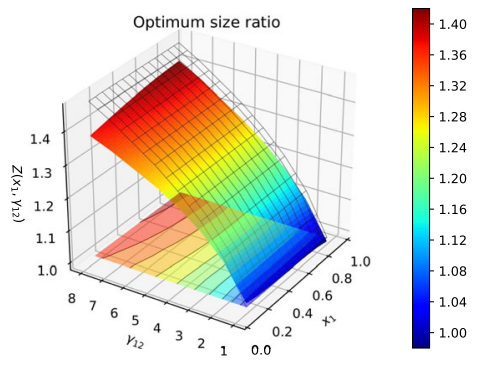

(c)

FIG. 4. (a) A realization of the optimal two-phase system at $\Gamma_{22}=5$ with volume fraction 0.15 . (b) The local volume-fraction variance $\sigma_{V}^{2}(R)$ as a function of the spherical observation window of radius $R$ for different size ratios (optimal value 1.3). The scaling behaviors for $R^{-2}$ (nonhyperuniform) and $R^{-3}$ (hyperuniform) are included to guide the eye. (c) Optimal size ratio $Z\left(x_{1}, \gamma_{12}\right)$ in terms of composition and coupling strength ratio, $\gamma_{12}$, as defined by Eq. (6).

$\int_{0}^{\infty}\left\{1-\exp \left[-u(r) / k_{B} T\right]\right\} d r \approx 1.354 \Gamma^{(1 / 3)} / \sqrt{\rho_{1}}$, we find a good empirical expression for the optimal ratio in this region: $Z\left(x_{1}, \gamma_{12}\right) \sim\left(\gamma_{12}{ }^{(1 / 3)}+\gamma_{12}{ }^{(1 / 6)}+1\right) / 3$, which is represented by the wireframe in Fig. 4(c). Using this figure, one may determine the experimental characteristics for our colloidal mixture to become effectively hyperuniform.

Generalizations.-Our protocol can be applied to other long-ranged interactions, dimensionalities, and/or polydispersity. Recall that to reach the key relation $\phi(k) \approx \pi$, we have not assumed anything about the exact form of the interaction, nor dimensionality, as long as the system is hard to compress. For polydisperse systems, incompressibility means that there exists an eigenvector of the structure factor matrix $S_{i j}$ which leads to a vanishing eigenvalue at infinite wavelength [32]. Thus, there exists a vector a such that

$$
\sum_{i, j} a_{i} S_{i j}\left(0^{+}\right) a_{j}=0
$$

To make the polydisperse system a hyperuniform twophase medium by decoration, note that the spectral density $\tilde{\chi}_{V}\left(0^{+}\right)$has the following form:

$$
\tilde{\chi}_{V}\left(0^{+}\right) \propto \sum_{i, j} \sqrt{x_{i}} V\left(R_{i}\right) S_{i j}\left(0^{+}\right) \sqrt{x_{j}} V\left(R_{j}\right),
$$

where $V\left(R_{i}\right)$ is the volume of a particle with radius $R_{i}$. Comparing Eq. (7) and Eq. (8) it immediately follows that the optimal composition has the property that $V\left(R_{i}\right) \propto$ $a_{i} / \sqrt{x_{i}}$, i.e., $R_{i} \propto a_{i}^{1 / d} / x_{i}{ }^{1 / 2 d}$. For the dipolar system we consider, one can verify that $a=\left[1 / \sqrt{S_{11}\left(0^{+}\right)}\right.$, $\left.1 / \sqrt{S_{22}\left(0^{+}\right)}\right]$is the eigenvector that we are looking for from Eq. (2). Plugging $a$ and $d=2$ into the scaling relation for $R_{i}$, we indeed recover relation Eq. (6). Simulation results given in the SM for a three-component system provide further confirmation of our methodology.
Furthermore, we note that our protocol can be generalized to treat random fields derived from particle configurations as well as hyperuniformity with respect to other types of fluctuations, e.g., by choosing $R_{1} / R_{2}=$ $\left[\rho_{2} S_{22}\left(0^{+}\right) / \rho_{1} S_{11}\left(0^{+}\right)\right]^{(1 / 2)}$, the binary system is hyperuniform with respect to interfacial-surface-area fluctuations [19]; see the SM for a detailed discussion.

In conclusion, we have proposed a highly feasible and robust equilibrium protocol that can be employed in the laboratory to fabricate large disordered hyperuniform materials, using binary paramagnetic colloidal particles confined in a 2D plane. The destructive interference of scattering events between the two species of the binary system at infinite wavelength enables us to design the optimal size ratio. Although the present work stresses 2D dipolar binary systems, our protocol turns out to be general and suitable to systems with other sufficiently long-ranged soft interactions, dimensionalities, and/or polydispersity. Our methodology opens up avenues to synthesize large, tunable photonic hyperuniform materials that function in the visible to infrared range and thus may accelerate the discovery of novel photonic materials.

The authors are grateful to C. Maher, T. Middlemas, and C. Likos for fruitful discussions. Z. M. and S. T. acknowledge the support of the National Science Foundation under Grant No. DMR-1714722. E. L. acknowledges the support from the Agencia Estatal de Investigacin and Fondo Europeo de Desarrollo Regional (FEDER) under Grant No. FIS2017-89361-C3-2-P and from European Unions Horizon 2020 Research and Innovation Staff Exchange programme under the Marie Skodowska-Curie Grant Agreement No. 734276.

*torquato@electron.princeton.edu

[1] S. Torquato and F. H. Stillinger, Phys. Rev. E 68, 041113 (2003). 
[2] F. J. Dyson, J. Math. Phys. (N.Y.) 3, 140 (1962).

[3] B. Jancovici, Phys. Rev. Lett. 46, 386 (1981).

[4] S. Torquato, G. Zhang, and F. H. Stillinger, Phys. Rev. X 5, 021020 (2015).

[5] A. Donev, F. H. Stillinger, and S. Torquato, Phys. Rev. Lett. 95, 090604 (2005).

[6] R. Kurita and E. R. Weeks, Phys. Rev. E 84, 030401(R) (2011).

[7] C. E. Zachary, Y. Jiao, and S. Torquato, Phys. Rev. E 83, 051308 (2011).

[8] Y. A. Gerasimenko, I. Vaskivskyi, M. Litskevich, J. Ravnik, J. Vodeb, M. Diego, V. Kabanov, and D. Mihailovic, Nat. Mater. 18, 1078 (2019).

[9] D. Hexner and D. Levine, Phys. Rev. Lett. 114, 110602 (2015)

[10] J. H. Weijs, R. Jeanneret, R. Dreyfus, and D. Bartolo, Phys. Rev. Lett. 115, 108301 (2015).

[11] T. Bertrand, D. Chatenay, and R. Voituriez, New J. Phys. 21, 123048 (2019).

[12] Q.-L. Lei and R. Ni, Proc. Natl. Acad. Sci. U.S.A. 116, 22983 (2019).

[13] Q.-L. Lei, M. P. Ciamarra, and R. Ni, Sci. Adv. 5, eaau7423 (2019).

[14] A. Chremos and J. F. Douglas, Phys. Rev. Lett. 121, 258002 (2018).

[15] Y. Jiao, T. Lau, H. Hatzikirou, M. Meyer-Hermann, J. C. Corbo, and S. Torquato, Phys. Rev. E 89, 022721 (2014).

[16] A. Mayer, V. Balasubramanian, T. Mora, and A. M. Walczak, Proc. Natl. Acad. Sci. U.S.A. 112, 5950 (2015).

[17] H. L. Montgomery, Proc. Symp. Pure Math 24, 181 (1973), http://www.ams.org/books/pspum/024/.

[18] S. Torquato, Phys. Rep. 745, 1 (2018).

[19] S. Torquato, Phys. Rev. E 94, 022122 (2016).

[20] S. Torquato, Random Heterogeneous Materials: Microstructure and Macroscopic Properties (Springer Science \& Business Media, New York, 2002).

[21] M. Florescu, S. Torquato, and P. J. Steinhardt, Proc. Natl. Acad. Sci. U.S.A. 106, 20658 (2009).

[22] W. Man, M. Florescu, E. P. Williamson, Y. He, S. R. Hashemizad, B. Y. Leung, D. R. Liner, S. Torquato, P. M. Chaikin, and P. J. Steinhardt, Proc. Natl. Acad. Sci. U.S.A. 110, 15886 (2013).

[23] J. Haberko, N. Muller, and F. Scheffold, Phys. Rev. A 88, 043822 (2013).

[24] C. De Rosa, F. Auriemma, C. Diletto, R. Di Girolamo, A. Malafronte, P. Morvillo, G. Zito, G. Rusciano, G. Pesce, and A. Sasso, Phys. Chem. Chem. Phys. 17, 8061 (2015).

[25] P. M. Piechulla, L. Muehlenbein, R. B. Wehrspohn, S. Nanz, A. Abass, C. Rockstuhl, and A. Sprafke, Adv. Opt. Mater. 6, 1701272 (2018).

[26] F. Bigourdan, R. Pierrat, and R. Carminati, Opt. Express 27, 8666 (2019).

[27] S. Gorsky, W. Britton, Y. Chen, J. Montaner, A. Lenef, M. Raukas, and L. Dal Negro, APL Photonics 4, 110801 (2019).
[28] V. Romero-García, N. Lamothe, G. Theocharis, O. Richoux, and L. M. García-Raffi, Phys. Rev. Applied 11, 054076 (2019).

[29] H. Noh, S. F. Liew, V. Saranathan, S. G. Mochrie, R. O. Prum, E. R. Dufresne, and H. Cao, Adv. Mater. 22, 2871 (2010).

[30] K. Chung, S. Yu, C.-J. Heo, J. W. Shim, S.-M. Yang, M. G. Han, H.-S. Lee, Y. Jin, S. Y. Lee, N. Park, and J. H. Shin, Adv. Mater. 24, 2375 (2012).

[31] J. Ricouvier, R. Pierrat, R. Carminati, P. Tabeling, and P. Yazhgur, Phys. Rev. Lett. 119, 208001 (2017).

[32] L. Berthier, P. Chaudhuri, C. Coulais, O. Dauchot, and P. Sollich, Phys. Rev. Lett. 106, 120601 (2011).

[33] J. H. Weijs and D. Bartolo, Phys. Rev. Lett. 119, 048002 (2017).

[34] S. Wilken, R. E. Guerra, D. J. Pine, and P. M. Chaikin, arXiv:2002.04499 [Phys. Rev. Lett. (to be published)].

[35] Z. Ma and S. Torquato, J. Appl. Phys. 121, 244904 (2017).

[36] M. Salvalaglio, M. Bouabdellaoui, M. Bollani, A. Benali, L. Favre, J.-B. Claude, J. Wenger, P. de Anna, F. Intonti, A. Voigt, and M. Abbarchi, arXiv:1912.02952.

[37] S. Atkinson, G. Zhang, A. B. Hopkins, and S. Torquato, Phys. Rev. E 94, 012902 (2016).

[38] D. Chen, E. Lomba, and S. Torquato, Phys. Chem. Chem. Phys. 20, 17557 (2018).

[39] F. Ebert, G. Maret, and P. Keim, Eur. Phys. J. E 29, 311 (2009).

[40] S. Z. Lin, B. Zheng, and S. Trimper, Phys. Rev. E 73, 066106 (2006).

[41] S. C. Kapfer and W. Krauth, Phys. Rev. Lett. 114, 035702 (2015).

[42] K. Zahn, R. Lenke, and G. Maret, Phys. Rev. Lett. 82, 2721 (1999).

[43] C. P. Kelleher, R. E. Guerra, A. D. Hollingsworth, and P. M. Chaikin, Phys. Rev. E 95, 022602 (2017).

[44] B. I. Halperin and D. R. Nelson, Phys. Rev. Lett. 41, 121 (1978).

[45] N. Hoffmann, C. N. Likos, and H. Löwen, J. Phys. Condens. Matter 18, 10193 (2006)

[46] N. Hoffmann, F. Ebert, C. N. Likos, H. Löwen, and G. Maret, Phys. Rev. Lett. 97, 078301 (2006).

[47] T. S. Grigera and G. Parisi, Phys. Rev. E 63, 045102(R) (2001).

[48] F. J. Rogers and D. A. Young, Phys. Rev. A 30, 999 (1984).

[49] E. Lomba, J. J. Weis, and S. Torquato, Phys. Rev. E 96, 062126 (2017).

[50] See Supplemental Material at http://link.aps.org/ supplemental/10.1103/PhysRevLett.125.068002 for additional information.

[51] N. Ashcroft and D. C. Langreth, Phys. Rev. 156, 685 (1967).

[52] S. A. Khrapak, N. P. Kryuchkov, and S. O. Yurchenko, Phys. Rev. E 97, 022616 (2018). 